\title{
AN EXPLICIT SOLVER TO THE DIRICHLET PROBLEM FOR THE LAPLACE EQUATION IN A DISK IN POLYNOMIALS
}

\author{
V. L. Borsch* I. E. Platonova ${ }^{\dagger}$
}

Communicated by Prof. V. Kapustyan

\begin{abstract}
Explicit formulas for the solution to the Dirichlet problem for the Laplace equation in a disk in polynomials are derived and discussed.
\end{abstract}

Key words: the Dirichlet problem, explicit solution in polynomials, degenerate elliptic equation.

2010 Mathematics Subject Classification: 31A25, 31B20, 35A09, 35G15, 35J25.

\section{Introduction to the solver}

Following [1,2], we consider the well known Dirichlet problem for the Laplace equation in a disk of radius $c$ centered at point $\boldsymbol{x}_{0}$ in the plane $\mathbb{R}^{2}$ parameterized by cartesian orthogonal coordinates $\boldsymbol{x}=\left(x_{1}, x_{2}\right)$

$$
\left\{\begin{aligned}
\Delta_{\boldsymbol{x}} u(\boldsymbol{x}) & =0, & \boldsymbol{x} & \in \mathcal{B}_{c}^{2}\left(\boldsymbol{x}_{0}\right):=\left\{\boldsymbol{x}:\left|\boldsymbol{x}-\boldsymbol{x}_{0}\right|^{2}<c^{2}\right\}, \\
u(\boldsymbol{x}) & =Q_{m}^{\prime}(\boldsymbol{x}), & & \boldsymbol{x} \in \mathcal{S}_{c}^{2}\left(\boldsymbol{x}_{0}\right):=\left\{\boldsymbol{x}:\left|\boldsymbol{x}-\boldsymbol{x}_{0}\right|^{2}=c^{2}\right\},
\end{aligned}\right.
$$

where the boundary function is a polynomial of degree $m$

$$
Q_{m}(\boldsymbol{x})=\sum_{p+q=0}^{m} a_{p, q} x_{1}^{p} x_{2}^{q}
$$

$p, q \in \mathbb{Z} \backslash \mathbb{Z}_{-}, a_{p, q} \in \mathbb{R}$, and prime means that the domain of definition of $Q_{m}(\boldsymbol{x})$ is restricted to $\mathcal{S}_{c}^{2}\left(\boldsymbol{x}_{0}\right)$.

The above problem is known [3] to have a unique solution, and the solution is evidently to be a polynomial $U_{m}(\boldsymbol{x})$ of degree $m$.

In [1] we proved the following

${ }^{*}$ Dept. of Differential Equations, Faculty of Mech \& Math, Oles Honchar Dnipro National University, 72, Gagarin av., Dnipro, 49010, Ukraine, bvl@dsu.dp.ua

${ }^{\dagger}$ College of Radioelectronics, 18, Schmidt st., Dnipro, 49006, Ukraine, irina.platonof $f @ m a i l . r u$

(C) V. L. Borsch, I. E. Platonova, 2019. 
Proposition 1.1. The solution to the Dirichlet problem (1.1) admits the following representation

$$
u(\boldsymbol{x})=U_{m}(\boldsymbol{x})=F_{2}(\boldsymbol{x}) P_{m-2}(\boldsymbol{x})+Q_{m}(\boldsymbol{x}),
$$

where $F_{2}(\boldsymbol{x})$ is the polynomial of degree 2 specifying the boundary of the disk: $F_{2}(\boldsymbol{x})=c^{2}-\left|\boldsymbol{x}-\boldsymbol{x}_{0}\right|^{2}=0$, and $P_{m-2}(\boldsymbol{x})$ is a uniquely determined polynomial of degree $m-2$.

The way of proving proposition 1.1 was based on the Fourier method. Then, in [2] we gave an other proof based on the Poisson integral formula for the solution. Representation (1.3) was illustrated in [1] by numerous examples where $c, \boldsymbol{x}_{0}$ and $P_{m}(\boldsymbol{x})$ were changed: first, we obtained solutions to (1.1) using the Fourier method and, second, showed representation (1.3) to hold by dividing polynomial solution $U_{m}(\boldsymbol{x})$ by polynomial $F_{2}(\boldsymbol{x})$ with remainder $Q_{m}(\boldsymbol{x})$. From this some shrewd readers of [1] concluded that the method we used to prove representation (1.3) gives no explicit formula for polynomial $P_{m-2}(\boldsymbol{x})$. Hence, in the current study we derive the proper explicit formulas for polynomial $P_{m-2}(\boldsymbol{x})$ literally following [1].

The article is arranged as follows.

As in [1], we apply the direct transformation of independent variables $\boldsymbol{x} \rightarrow \boldsymbol{y}$ : $\boldsymbol{x}=\boldsymbol{y}+\boldsymbol{x}_{0}$, and replace the original Dirichlet problem (1.1) with the following derived one

$$
\left\{\begin{aligned}
\Delta_{\boldsymbol{y}} w(\boldsymbol{y}) & =0, & & \boldsymbol{y} \in \mathcal{B}_{c}^{2}(\mathbf{0}), \\
w(\boldsymbol{y}) & =R_{m}^{\prime}(\boldsymbol{y}), & & \boldsymbol{y} \in \mathcal{S}_{c}^{2}(\mathbf{0}),
\end{aligned}\right.
$$

where the disk is centered at the origin of cartesian coordinates $\boldsymbol{y}=\left(y_{1}, y_{2}\right)$, $w(\boldsymbol{y}):=u\left(\boldsymbol{y}+\boldsymbol{x}_{0}\right)$, and

$$
R_{m}(\boldsymbol{y}):=Q_{m}\left(\boldsymbol{y}+\boldsymbol{x}_{0}\right)=\sum_{p+q=0}^{m} b_{p, q} y_{1}^{p} y_{2}^{q}=\sum_{p+q=0}^{m} b_{p, q} R_{p, q}(\boldsymbol{y})
$$

Representation (1.3) for the solution to the derived Dirichlet problem reads

$$
w(\boldsymbol{y})=W_{m}(\boldsymbol{y})=G_{2}(\boldsymbol{y}) S_{m-2}(\boldsymbol{y})+R_{m}(\boldsymbol{y}),
$$

where $G_{2}(\boldsymbol{y}):=F_{2}\left(\boldsymbol{y}+\boldsymbol{x}_{0}\right)=c^{2}-|\boldsymbol{y}|^{2}, S_{m-2}(\boldsymbol{y}):=P_{m-2}\left(\boldsymbol{y}+\boldsymbol{x}_{0}\right)$.

Then, in Section 2 we consider the contributions

$$
W_{p, q}(\boldsymbol{y})=G_{2}(\boldsymbol{y}) S_{p, q}(\boldsymbol{y})+R_{p, q}(\boldsymbol{y})
$$


of the monomials $R_{p, q}(\boldsymbol{y})=y_{1}^{p} y_{2}^{q}$ of the boundary polynomial $R_{m}(\boldsymbol{y})$ (1.5) to the solution $W_{m}(\boldsymbol{y})(1.6)$, where

$$
\begin{gathered}
W_{m}(\boldsymbol{y})=\sum_{p+q=0}^{m} W_{p, q}(\boldsymbol{y}), \\
S_{m-2}(\boldsymbol{y})=\sum_{p+q=0}^{m} b_{p, q} S_{p, q}(\boldsymbol{y}),
\end{gathered}
$$

$W_{p, q}(\boldsymbol{y})$ and $S_{p, q}(\boldsymbol{y})$ are polynomials of degree $p+q$ and $p+q-2$ respectively. To find the polynomials $S_{p, q}(\boldsymbol{y})$ we use the Fourier method. For representation (1.3) for the solution to the original Dirichlet problem to be obtained one should apply the inverse transformation of independent variables $\boldsymbol{y} \rightarrow \boldsymbol{x}: \boldsymbol{y}=$ $\boldsymbol{x}-\boldsymbol{x}_{0}$.

In Section 3 we illustrate the resulted explicit formulas of Section 2 for $S_{p, q}(\boldsymbol{y})$ by numerous examples.

In Section 4 we show how to simplify the resulted explicit formulas for $S_{p, q}(\boldsymbol{y})$ and eventually how to find polynomial $S_{m-2}(\boldsymbol{y})$ not treating the monomials $R_{p, q}(\boldsymbol{y})$ separately.

In Section 5 we give supplementary data for readers to check propositions of Section 4 .

In Section 6 we discuss briefly some other methods to find polynomials $S_{p, q}(\boldsymbol{y})$.

In Section 7 we show in what way other methods could help us obtain polynomials $S_{p, q}(\boldsymbol{y})$.

\section{The Fourier method to find $S_{p, q}(\boldsymbol{y})$}

Before applying the well known Fourier method [3] to derive explicit formulas for representation (1.6), we give a brief description of the method to clarify the main idea we utilized in [1].

Let a boundary monomial $R_{p, q}^{\prime}(p+q \geqslant 3$ and is odd $)$ to have the following Fourier series (possible cases are presented in Tbl. 1 at p. 83)

$$
\stackrel{\circ}{R}_{p, q}^{\prime}(\varphi)=c^{p+q} \sum_{\mu=0}^{\frac{p+q-1}{2}} a_{2 \mu+1} \cos [(2 \mu+1) \varphi],
$$

then extension of the boundary monomial to disk $\overline{\mathcal{B}_{c}^{2}(\mathbf{0})}$ is evidently to be

$$
\stackrel{\circ}{R}_{p, q}^{\prime}(\varphi)=r^{p+q} \sum_{\mu=0}^{\frac{p+q-1}{2}} a_{2 \mu+1} \cos [(2 \mu+1) \varphi]
$$

and the solution to the Dirichlet problem is as follows 


$$
\stackrel{\circ}{W}_{p, q}(r, \varphi)=c^{p+q} \sum_{\mu=0}^{\frac{p+q-1}{2}}\left(\frac{r}{c}\right)^{2 \mu+1} a_{2 \mu+1} \cos [(2 \mu+1) \varphi],
$$

where the circle over the function name indicates changing cartesian coordinates to polar ones: $y_{1}=r \cos \varphi, y_{2}=r \sin \varphi$.

Then we transform the solution identically as

$$
\begin{aligned}
\stackrel{\circ}{W}_{p, q}(r, \varphi) & =\stackrel{\circ}{W}_{p, q}(r, \varphi)-\stackrel{\circ}{R}_{p, q}(r, \varphi)+\stackrel{\circ}{R}_{p, q}(r, \varphi) \\
& =\sum_{\mu=1}^{\frac{p+q-1}{2}}\left[c^{p+q}\left(\frac{r}{c}\right)^{2 \mu+1}-r^{p+q}\right] a_{2 \mu+1} \cos [(2 \mu+1) \varphi]+\stackrel{\circ}{R}_{p, q}(r, \varphi)
\end{aligned}
$$

and manipulate the expression in brackets algebraically (provided $2 \mu+1<p+q$ and $5 \leqslant p+q)$

$$
\begin{aligned}
. c^{p+q}\left(\frac{r}{c}\right)^{2 \mu+1}-r^{p+q} & =c^{p+q-2 \mu-1} r^{2 \mu+1}-r^{p+q} \\
& =c^{p+q-2 \mu-1} r^{2 \mu+1}-r^{p+q-2 \mu-1} r^{2 \mu+1} \\
& =r^{2 \mu+1}\left(c^{p+q-2 \mu-1}-r^{p+q-2 \mu-1}\right) \\
& =r^{2 \mu+1}\left(c^{2}-r^{2}\right) A_{p+q-2 \mu-3}(c, r),
\end{aligned}
$$

where homogeneous in $c$ and $r$ polynomials are defined as

$$
A_{2 k}(c, r)=\left\{\begin{aligned}
1, & k=0, \\
c^{2 k}+c^{2 k-2} r^{2}+\ldots+c^{2} r^{2 k-2}+r^{2 k}, & k>1 .
\end{aligned}\right.
$$

Eventually we obtain the required representation as

$$
\begin{aligned}
\stackrel{\circ}{W}_{p, q}(r, \varphi) & =\left(c^{2}-r^{2}\right) \sum_{\mu=1}^{\frac{p+q-1}{2}-1} a_{2 \mu+1} A_{p+q-2 \mu-3}(c, r) r^{2 \mu+1} \cos [(2 \mu+1) \varphi]+\stackrel{\circ}{R}_{p, q}(r, \varphi) \\
& =\left(c^{2}-r^{2}\right) \sum_{\mu=1}^{\frac{p+q-1}{2}-1} a_{2 \mu+1} A_{p+q-2 \mu-3}(c, r) H_{2 \mu+1,1}(r, \varphi)+\stackrel{\circ}{R}_{p, q}(r, \varphi),
\end{aligned}
$$

where first there appear harmonic polynomials [8]

$$
\stackrel{\circ}{H}_{k, 1}(r, \varphi)=r^{k} \cos k \varphi, \quad \stackrel{\circ}{H}_{k, 2}(r, \varphi)=r^{k} \sin k \varphi .
$$


To obtain the Fourier series of boundary monomials $R_{p, q}$ we use well known formulas [4] for powers of trigonometric functions cos and sin in terms of these functions of multiples of the argument

$$
\left\{\begin{array}{l}
\cos ^{p} \varphi=\frac{1}{2^{p-1}} \sum_{\mu=0}^{\frac{p-1}{2}} C_{p}^{\mu} \cos \varphi_{\mu} \\
\sin ^{q} \varphi=\frac{1}{2^{q-1}} \sum_{\gamma=0}^{\frac{q-1}{2}} C_{q}^{\gamma} \sin \varphi_{\gamma}(-1)^{\frac{q-1}{2}+\gamma}
\end{array}\right.
$$

when $p$ and $q$ are odd integers, and

$$
\left\{\begin{array}{l}
\cos ^{p} \varphi=\frac{1}{2^{p}} C_{p}^{\frac{p}{2}}+\frac{1}{2^{p-1}} \sum_{\mu=0}^{\frac{p}{2}-1} C_{p}^{\mu} \cos \varphi_{\mu}, \\
\sin ^{q} \varphi=\frac{1}{2^{q}} C_{q}^{\frac{q}{2}}+\frac{1}{2^{q-1}} \sum_{\gamma=0}^{\frac{q}{2}-1} C_{q}^{\gamma} \cos \varphi_{\gamma}(-1)^{\frac{q}{2}+\gamma},
\end{array}\right.
$$

when $p$ and $q$ are even integers; and $\varphi_{\mu}:=p_{\mu} \varphi=(p-2 \mu) \varphi, \varphi_{\gamma}:=q_{\gamma} \varphi=(q-$ $2 \gamma) \varphi$, and formulas

$$
\left\{\begin{array}{l}
2 \cos \varphi_{1} \cos \varphi_{2}=\cos \left(\varphi_{1}-\varphi_{2}\right)+\cos \left(\varphi_{1}+\varphi_{2}\right), \\
2 \sin \varphi_{1} \cos \varphi_{2}=\sin \left(\varphi_{1}-\varphi_{2}\right)+\sin \left(\varphi_{1}+\varphi_{2}\right)
\end{array}\right.
$$

To transform harmonic polynomials (2.2) to cartesian variables we use well known formulas [4] for trigonometric functions cos and sin of multiples of the argument in terms of powers of these functions (for $p, q$ being odd and even respectively)

$$
\left\{\begin{array}{l}
\cos p \varphi=\sum_{\mu=0}^{\frac{p-1}{2}}(-1)^{\mu} C_{p}^{2 \mu} \cos ^{p-2 \mu} \varphi \sin ^{2 \mu} \varphi \\
\sin q \varphi=\sum_{\gamma=0}^{\frac{q-1}{2}}(-1)^{\gamma} C_{q}^{2 \gamma+1} \cos ^{q-2 \gamma-1} \varphi \sin ^{2 \gamma+1} \varphi
\end{array}\right.
$$




$$
\left\{\begin{array}{l}
\cos p \varphi=\sum_{\mu=0}^{\frac{p}{2}}(-1)^{\mu} C_{p}^{2 \mu} \cos ^{p-2 \mu} \varphi \sin ^{2 \mu} \varphi \\
\sin q \varphi=\sum_{\gamma=0}^{\frac{q}{2}-1}(-1)^{\gamma} C_{q}^{2 \gamma+1} \cos ^{q-2 \gamma-1} \varphi \sin ^{2 \gamma+1} \varphi .
\end{array}\right.
$$

\subsection{Trivial cases}

We call trivial those cases when the boundary monomials are harmonic ones of degree 1 and 2: a) $p=1, q=0$; b) $p=0, q=1$; c) $p=1, q=1$. Then polynomials $S_{1,0}, S_{0,1}$ and $S_{1,1}$ are evidently to equal zero identically.

\subsection{Other missing cases}

Other missing cases are as follows: a) $p \geqslant 3$ is odd and $q=0$; b) $p=0$ and $q>1$ is odd; c) $p \geqslant 2$ is even and $q=0$; d) $p=0$ and $q \geqslant 2$ is even. They are thoroughly studied or discussed in [1].

2.3. $p \geqslant 1$ is odd and $q \geqslant 1$ is odd, $p+q \geqslant 4$

First we find the Fourier series for the given boundary monomial restricted to $\mathcal{S}_{c}^{2}(\mathbf{0})$

$$
\begin{aligned}
\stackrel{\circ}{R}_{p, q}^{\prime}(\varphi) & =c^{p} \cos ^{p} \varphi c^{q} \sin ^{q} \varphi \\
& =\frac{c^{p+q}}{2^{p+q-2}}\left(\sum_{\mu=0}^{\frac{p-1}{2}} C_{p}^{\mu} \cos \varphi_{\mu}\right)\left(\sum_{\gamma=0}^{\frac{q-1}{2}}(-1)^{\frac{q-1}{2}+\gamma} C_{q}^{\gamma} \sin \varphi_{\gamma}\right) \\
& =\frac{c^{p+q}}{2^{p+q-1}} \sum_{\mu=0}^{\frac{p-1}{2}} \sum_{\gamma=0}^{\frac{q-1}{2}}(-1)^{\frac{q-1}{2}+\gamma} C_{p}^{\mu} C_{q}^{\gamma} \sin \left(\varphi_{\gamma}+\varphi_{\mu}\right) \\
& +\frac{c^{p+q}}{2^{p+q-1}} \sum_{\mu=0}^{\frac{p-1}{2}} \sum_{\gamma=0}^{\frac{q-1}{2}}(-1)^{\frac{q-1}{2}+\gamma} C_{p}^{\mu} C_{q}^{\gamma} \sin \left(\varphi_{\gamma}-\varphi_{\mu}\right) .
\end{aligned}
$$

Then we extend the boundary monomial to $\overline{\mathcal{B}_{c}^{2}(\mathbf{0})}$

$$
\begin{aligned}
\stackrel{\circ}{R}_{p, q}(r, \varphi) & =\frac{r^{p+q}}{2^{p+q-1}} \sum_{\mu=0}^{\frac{p-1}{2}} \sum_{\gamma=0}^{\frac{q-1}{2}}(-1)^{\frac{q-1}{2}+\gamma} C_{p}^{\mu} C_{q}^{\gamma} \sin \left(\varphi_{\gamma}+\varphi_{\mu}\right) \\
& +\frac{r^{p+q}}{2^{p+q-1}} \sum_{\mu=0}^{\frac{p-1}{2}} \sum_{\gamma=0}^{\frac{q-1}{2}}(-1)^{\frac{q-1}{2}+\gamma} C_{p}^{\mu} C_{q}^{\gamma} \sin \left(\varphi_{\gamma}-\varphi_{\mu}\right)
\end{aligned}
$$


set up the solution to the Dirichlet problem following the Fourier method and apply the identical transformation discussed in Section 2

$$
\begin{aligned}
\stackrel{\circ}{W}_{p, q}(r, \varphi) & =\frac{1}{2^{p+q-1}} \underbrace{\sum_{\mu=0}^{\frac{p-1}{2}} \sum_{\gamma=0}^{\frac{q-1}{2}}}_{\mu+\gamma>0}(-1)^{\frac{q-1}{2}+\gamma} C_{p}^{\mu} C_{q}^{\gamma}\left[c^{p+q}\left(\frac{r}{c}\right)^{q_{\gamma}+p_{\mu}}-r^{p+q}\right] \sin \left(\varphi_{\gamma+\mu}\right) \\
& +\frac{1}{2^{p+q-1}} \underbrace{\sum_{\mu=0}^{\frac{p-1}{2}} \sum_{\gamma=0}^{\frac{q-1}{2}}}_{p_{\mu}<q_{\gamma}}(-1)^{\frac{q-1}{2}+\gamma} C_{p}^{\mu} C_{q}^{\gamma}\left[c^{p+q}\left(\frac{r}{c}\right)^{q_{\gamma}-p_{\mu}}-r^{p+q}\right] \sin \left(\varphi_{\gamma-\mu}\right) \\
& -\frac{1}{2^{p+q-1}} \underbrace{\frac{p-1}{2} \sum_{\gamma=0}^{\frac{q-1}{2}}}_{p_{\mu}>q_{\gamma}}(-1)^{\frac{q-1}{2}+\gamma} C_{p}^{\mu} C_{q}^{\gamma}\left[c^{p+q}\left(\frac{r}{c}\right)^{q_{\gamma}-p_{\mu}}-r^{p+q}\right] \sin \left(\varphi_{\mu-\gamma}\right) \\
& +\stackrel{\circ}{R}_{p, q}(r, \varphi),
\end{aligned}
$$

where the following auxiliary notation is used: $\varphi_{\gamma+\mu}=\varphi_{\gamma}+\varphi_{\mu}, \varphi_{\gamma-\mu}=\varphi_{\gamma}-\varphi_{\mu}$, $\varphi_{\mu-\gamma}=\varphi_{\mu}-\varphi_{\gamma}$.

After some algebraic manipulations discussed and explained in Section 2 we present the polynomial $S_{p, q}$ in polar variables separately

$$
\begin{aligned}
2^{p+q-1} \stackrel{\stackrel{S}{S}}{p, q}_{(r, \varphi)=} & \underbrace{\frac{p-1}{2} \frac{q-1}{2}}_{\mu=\gamma>0}(-1)^{\frac{q-1}{2}+\gamma} C_{p}^{\mu} C_{q}^{\gamma} A_{2(\mu+\gamma)-2}(c, r) r^{p_{\mu}+q_{\gamma}} \sin \left[\left(q_{\gamma}+p_{\mu}\right) \varphi\right] \\
& +\underbrace{\sum_{\mu=0}^{\frac{p-1}{2}} \sum_{\gamma=0}^{\frac{q-1}{2}}}_{p_{\mu}<q_{\gamma}}(-1)^{\frac{q-1}{2}+\gamma} C_{p}^{\mu} C_{q}^{\gamma} A_{2(p+\gamma-\mu)-2}(c, r) r^{q_{\gamma}-p_{\mu}} \sin \left[\left(q_{\gamma}-p_{\mu}\right) \varphi\right] \\
& -\underbrace{\sum_{\mu=0}^{\frac{p-1}{2}} \frac{q-1}{2}}_{p_{\mu}>q_{\gamma}}(-1)^{\frac{q-1}{2}+\gamma} C_{p}^{\mu} C_{q}^{\gamma} A_{2(q+\mu-\gamma)-2}(c, r) r^{p_{\mu}-q_{\gamma}} \sin \left[\left(p_{\mu}-q_{\gamma}\right) \varphi\right] .
\end{aligned}
$$

Transforming independent variables from cartesian to polar ones we eventually find polynomial $S_{p, q}$ 


$$
\begin{aligned}
2^{p+q-1} S_{p, q}(\boldsymbol{y}) & =\underbrace{\sum_{\mu=0}^{\frac{p-1}{2}} \sum_{\gamma=0}^{\frac{q-1}{2}}}_{\mu+\gamma>0}(-1)^{\frac{q-1}{2}+\gamma} C_{p}^{\mu} C_{q}^{\gamma} A_{2(\mu+\gamma)-2}(c,|\boldsymbol{y}|) H_{p+q-2\left(\mu_{\gamma}\right), 2}(\boldsymbol{y}) \\
& +\underbrace{\sum_{\mu=0}^{\frac{p-1}{2}} \sum_{\gamma=0}^{\frac{q-1}{2}}(-1)^{\frac{q-1}{2}+\gamma} C_{p}^{\mu} C_{q}^{\gamma} A_{2(p+\gamma-\mu)-2}(c,|\boldsymbol{y}|) H_{q-p-2(\gamma-\mu), 2}(\boldsymbol{y})}_{p_{\mu}<q_{\gamma}} \\
& -\underbrace{\sum_{\mu=0}^{\frac{p-1}{2}} \frac{q-1}{2}}_{p_{\mu}>q_{\gamma}}(-1)^{\frac{q-1}{2}+\gamma} C_{p}^{\mu} C_{q}^{\gamma} A_{2(q+\mu-\gamma)-2}(c,|\boldsymbol{y}|) H_{p-q-2(\mu-\gamma), 2}(\boldsymbol{y})
\end{aligned}
$$

2.4. $p \geqslant 1$ is odd and $q \geqslant 2$ is even, $p+q \geqslant 3$

We again start considering new case with finding the Fourier series for the given boundary monomial restricted to $\mathcal{S}_{c}^{2}(\mathbf{0})$

$$
\begin{aligned}
\stackrel{\circ}{R}_{p, q}^{\prime}(\varphi) & =c^{p} \cos ^{p} \varphi c^{q} \sin ^{q} \varphi \\
& =c^{p+q}\left(\frac{1}{2^{p-1}} \sum_{\mu=0}^{\frac{p-1}{2}} C_{p}^{\mu} \cos \varphi_{\mu}\right)\left(\frac{1}{2^{q}} C_{q}^{\frac{q}{2}}+\frac{1}{2^{q-1}} \sum_{\gamma=0}^{\frac{q}{2}-1}(-1)^{\frac{q}{2}+\gamma} C_{q}^{\gamma} \sin \varphi_{\gamma}\right) \\
& =\frac{c^{p+q}}{2^{p+q-1}} C_{q}^{\frac{q}{2}} \sum_{\mu=0}^{\frac{p-1}{2}} C_{p}^{\mu} \cos \varphi_{\mu} \\
& +\frac{c^{p+q}}{2^{p+q-1}} \sum_{\mu=0}^{\frac{p-1}{2}} \sum_{\gamma=0}^{\frac{q}{2}-1}(-1)^{\frac{q}{2}+\gamma} C_{p}^{\mu} C_{q}^{\gamma} \cos \left(\varphi_{\mu}+\varphi_{\gamma}\right) \\
& +\frac{c^{p+q}}{2^{p+q-1}} \sum_{\mu=0}^{\frac{p-1}{2}} \sum_{\gamma=0}^{\frac{q}{2}-1}(-1)^{\frac{q}{2}+\gamma} C_{p}^{\mu} C_{q}^{\gamma} \cos \left(\varphi_{\mu}-\varphi_{\gamma}\right) .
\end{aligned}
$$

Then as usually we extend the boundary monomial to the disk 


$$
\begin{aligned}
\stackrel{\circ}{R}_{p, q}^{\prime}(\varphi) & =\frac{r^{p+q}}{2^{p+q-1}} C_{q}^{\frac{q}{2}} \sum_{\mu=0}^{\frac{p-1}{2}} C_{p}^{\mu} \cos \varphi_{\mu} \\
& +\frac{r^{p+q}}{2^{p+q-1}} \sum_{\mu=0}^{\frac{p-1}{2}} \sum_{\gamma=0}^{\frac{q}{2}-1}(-1)^{\frac{q}{2}+\gamma} C_{p}^{\mu} C_{q}^{\gamma} \cos \left(\varphi_{\mu}+\varphi_{\gamma}\right) \\
& +\frac{r^{p+q}}{2^{p+q-1}} \sum_{\mu=0}^{\frac{p-1}{2}} \sum_{\gamma=0}^{\frac{q}{2}-1}(-1)^{\frac{q}{2}+\gamma} C_{p}^{\mu} C_{q}^{\gamma} \cos \left(\varphi_{\mu}-\varphi_{\gamma}\right),
\end{aligned}
$$

set up the solution to the problem and implement the identical transformation as follows

$$
\begin{aligned}
& \stackrel{\circ}{W}_{p, q}(r, \varphi)=\frac{1}{2^{p+q-1}} C_{q}^{\frac{q}{2}} \sum_{\mu=0}^{\frac{p-1}{2}} C_{p}^{\mu}\left[c^{p+q}\left(\frac{r}{c}\right)^{p_{\mu}}-r^{p+q}\right] \cos \varphi_{\mu}
\end{aligned}
$$

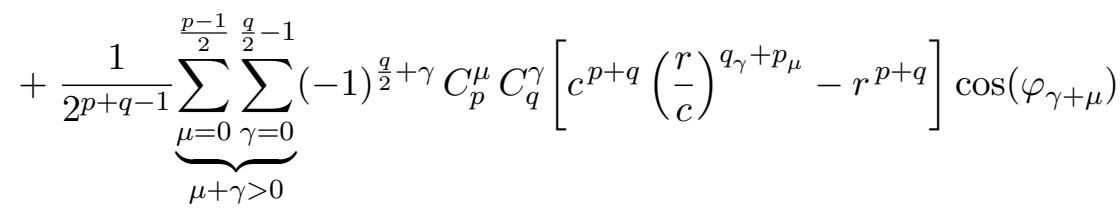

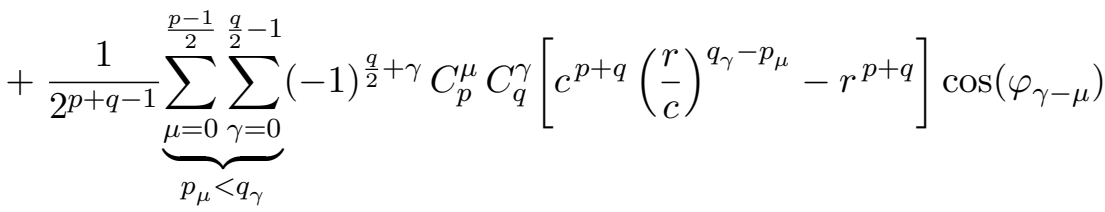

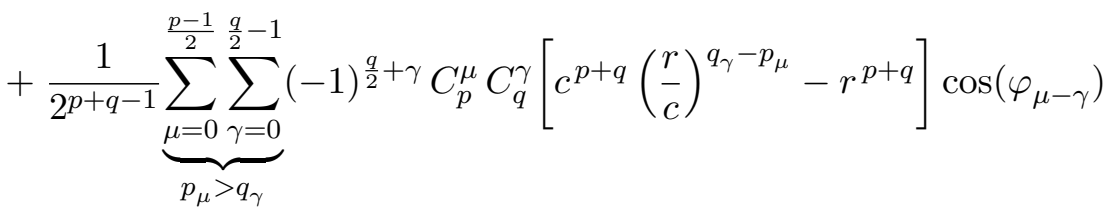

$$
\begin{aligned}
& +\stackrel{\circ}{R}_{p, q}(r, \varphi) \text {. }
\end{aligned}
$$

Now the polynomial $S_{p, q}$ has been ready to be presented in polar variables 


$$
\begin{aligned}
& 2^{p+q-1} \stackrel{\circ}{S}_{p, q}(r, \varphi)=C_{q}^{\frac{q}{2}} \sum_{\mu=0}^{\frac{p-1}{2}} C_{p}^{\mu} A_{q+2 \mu-2}(c, r) r^{p_{\mu}} \cos p_{\mu} \varphi
\end{aligned}
$$

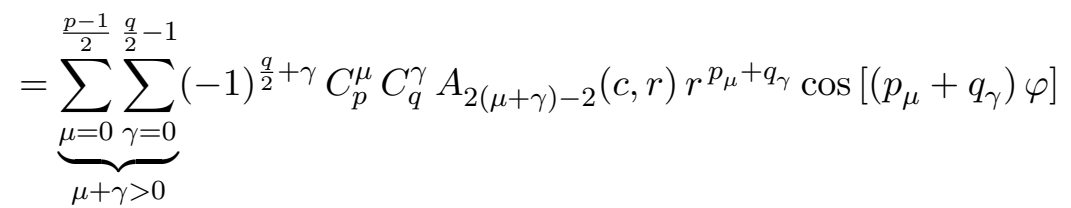

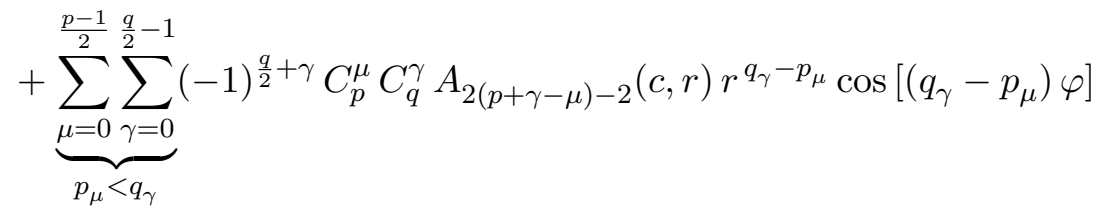

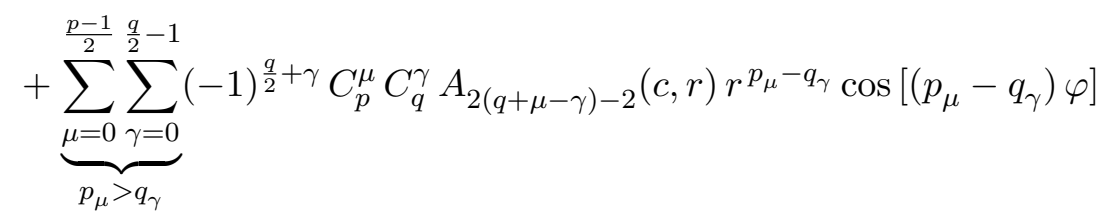

and finally in cartesian ones

$$
\begin{aligned}
2^{p+q-1} S_{p, q}(\boldsymbol{y}) & =C_{q}^{\frac{q}{2}} \sum_{\mu=0}^{\frac{p-1}{2}} C_{p}^{\mu} A_{q+2 \mu-2}(c,|\boldsymbol{y}|) H_{p-2 \mu, 1}(\boldsymbol{y}) \\
& =\underbrace{\sum_{\mu=0}^{\frac{p-1}{2}} \sum_{\gamma=0}^{\frac{q}{2}-1}(-1)^{\frac{q}{2}+\gamma} C_{p}^{\mu} C_{q}^{\gamma} A_{2(\mu+\gamma)-2}(c,|\boldsymbol{y}|) H_{p+q-2(\mu+\gamma), 1}(\boldsymbol{y})}_{\mu=\gamma>0} \\
& +\underbrace{\sum_{\mu=0}^{\frac{p-1}{2}} \sum_{\gamma=0}^{\frac{q}{2}-1}(-1)^{\frac{q}{2}+\gamma} C_{p}^{\mu} C_{q}^{\gamma} A_{2(p+\gamma-\mu)-2}(c,|\boldsymbol{y}|) H_{q-p-2(\gamma-\mu), 1}(\boldsymbol{y})}_{p_{\mu}<q_{\gamma}} \\
& +\underbrace{\sum_{\mu=0}^{\frac{p-1}{2}} \sum_{\gamma=0}^{\frac{q}{2}-1}}_{p_{\mu}>q_{\gamma}}(-1)^{\frac{q}{2}+\gamma} C_{p}^{\mu} C_{q}^{\gamma} A_{2(q+\mu-\gamma)-2}(c,|\boldsymbol{y}|) H_{p-q-2(\mu-\gamma), 1}(\boldsymbol{y}) .
\end{aligned}
$$


2.5. $p \geqslant 2$ is even and $q \geqslant 1$ is odd, $p+q \geqslant 3$

The Fourier series for the boundary monomial

$$
\begin{aligned}
\stackrel{\circ}{R}_{p, q}^{\prime}(\varphi) & =c^{p} \cos ^{p} \varphi c^{q} \sin ^{q} \varphi \\
& =c^{p+q}\left(\frac{1}{2^{p}} C_{p}^{\frac{p}{2}}+\frac{1}{2^{p-1}} \sum_{\mu=0}^{\frac{p}{2}-1} C_{p}^{\mu} \cos \varphi_{\mu}\right)\left(\frac{1}{2^{q-1}} \sum_{\gamma=0}^{\frac{q-1}{2}}(-1)^{\frac{q-1}{2}+\gamma} C_{q}^{\gamma} \sin \varphi_{\gamma}\right) \\
& =\frac{c^{p+q}}{2^{p+q-1}} C_{p}^{\frac{p}{2}} \sum_{\gamma=0}^{\frac{q-1}{2}}(-1)^{\frac{q-1}{2}+\gamma} C_{q}^{\gamma} \sin \varphi_{\gamma} \\
& +\frac{c^{p+q}}{2^{p+q-1}} \sum_{\mu=0}^{\frac{p}{2}-1} \sum_{\gamma=0}^{\frac{q-1}{2}}(-1)^{\frac{q-1}{2}+\gamma} C_{p}^{\mu} C_{q}^{\gamma} \sin \left(\varphi_{\gamma}+\varphi_{\mu}\right) \\
& +\frac{c^{p+q}}{2^{p+q-1}} \sum_{\mu=0}^{\frac{p}{2}-1} \sum_{\gamma=0}^{\frac{q-1}{2}}(-1)^{\frac{q-1}{2}+\gamma} C_{p}^{\mu} C_{q}^{\gamma} \sin \left(\varphi_{\gamma}-\varphi_{\mu}\right)
\end{aligned}
$$

is uniquely extended to the disk as the following function of polar variables

$$
\begin{aligned}
\stackrel{\circ}{R}_{p, q}(r, \varphi) & =\frac{r^{p+q}}{2^{p+q-1}} C_{p}^{\frac{p}{2}} \sum_{\gamma=0}^{\frac{q-1}{2}}(-1)^{\frac{q-1}{2}+\gamma} C_{q}^{\gamma} \sin \varphi_{\gamma} \\
& +\frac{r^{p+q}}{2^{p+q-1}} \sum_{\mu=0}^{\frac{p}{2}-1} \sum_{\gamma=0}^{\frac{q-1}{2}}(-1)^{\frac{q-1}{2}+\gamma} C_{p}^{\mu} C_{q}^{\gamma} \sin \left(\varphi_{\gamma}+\varphi_{\mu}\right) \\
& +\frac{r^{p+q}}{2^{p+q-1}} \sum_{\mu=0}^{\frac{p}{2}-1} \sum_{\gamma=0}^{\frac{q-1}{2}}(-1)^{\frac{q-1}{2}+\gamma} C_{p}^{\mu} C_{q}^{\gamma} \sin \left(\varphi_{\gamma}-\varphi_{\mu}\right) .
\end{aligned}
$$

Then the solution to the problem is set up as follows 


$$
\begin{aligned}
\stackrel{\circ}{W}_{p, q}(r, \varphi) & =\frac{1}{2^{p+q-1}} C_{p}^{\frac{p}{2}} \sum_{\gamma=0}^{\frac{q-1}{2}}(-1)^{\frac{q-1}{2}+\gamma} C_{q}^{\gamma}\left[c^{p+q}\left(\frac{r}{c}\right)^{q_{\gamma}}-r^{p+q}\right] \sin \varphi_{\gamma} \\
& +\frac{1}{2^{p+q-1}} \underbrace{\frac{p}{2}-1}_{\mu=0} \sum_{\mu=0}^{\frac{q-1}{2}}(-1)^{\frac{q-1}{2}+\gamma} C_{p}^{\mu} C_{q}^{\gamma}\left[c^{p+q}\left(\frac{r}{c}\right)^{q_{\gamma}+p_{\mu}}-r^{p+q}\right] \sin \left(\varphi_{\gamma+\mu}\right) \\
& +\frac{1}{2^{p+q-1}} \underbrace{\sum_{\mu=0}^{\frac{p}{2}-1} \sum_{\gamma=0}^{\frac{q-1}{2}}}_{p_{\mu}<q_{\gamma}}(-1)^{\frac{q-1}{2}+\gamma} C_{p}^{\mu} C_{q}^{\gamma}\left[c^{p+q}\left(\frac{r}{c}\right)^{q_{\gamma}-p_{\mu}}-r^{p+q}\right] \sin \left(\varphi_{\gamma-\mu}\right) \\
& -\frac{1}{2^{p+q-1}} \underbrace{\sum_{\mu=0}^{\frac{p}{2}-1} \sum_{\gamma=0}^{\frac{q-1}{2}}}_{p_{\mu}>q_{\gamma}}(-1)^{\frac{q-1}{2}+\gamma} C_{p}^{\mu} C_{q}^{\gamma}\left[c^{p+q}\left(\frac{r}{c}\right)^{p_{\mu}-q_{\gamma}}-r^{p+q}\right] \sin \left(\varphi_{\mu-\gamma}\right) \\
& +\stackrel{\circ}{R}_{p, q}(r, \varphi),
\end{aligned}
$$

from where the required polynomial is obtained in polar

$$
\begin{aligned}
& 2^{p+q-1} \stackrel{\circ}{S}_{p, q}(r, \varphi)=C_{p}^{\frac{p}{2}} \sum_{\gamma=0}^{\frac{q-1}{2}}(-1)^{\frac{q-1}{2}+\gamma} C_{q}^{\gamma} A_{p+2 \gamma-2}(c, r) r^{q_{\gamma}} \sin q_{\gamma} \varphi
\end{aligned}
$$

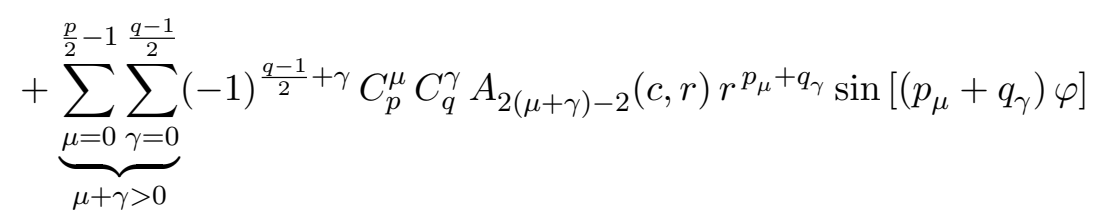

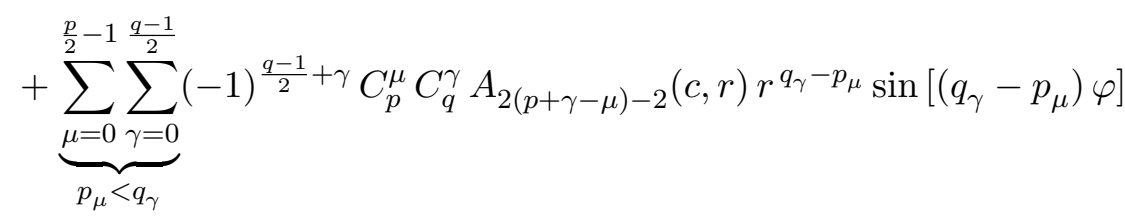

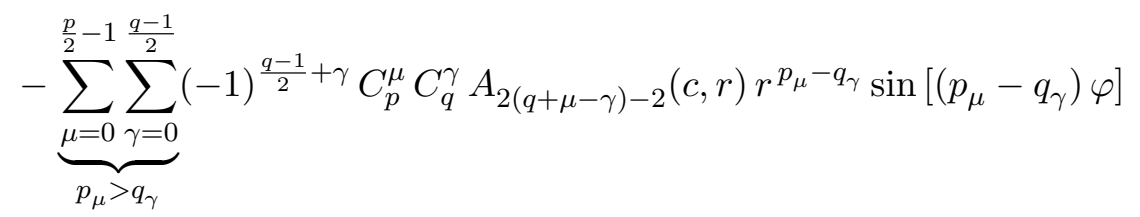

and then in cartesian variables 


$$
\begin{aligned}
2^{p+q-1} S_{p, q}(\boldsymbol{y}) & =C_{p}^{\frac{p}{2}} \sum_{\gamma=0}^{\frac{q-1}{2}}(-1)^{\frac{q-1}{2}+\gamma} C_{q}^{\gamma} A_{p+2 \gamma-2}(c,|\boldsymbol{y}|) H_{q-2 \gamma, 1}(\boldsymbol{y}) \\
& +\underbrace{\sum_{\mu=0}^{\frac{p}{2}-1} \sum_{\gamma=0}^{\frac{q-1}{2}}}_{\mu=0}(-1)^{\frac{q-1}{2}+\gamma} C_{p}^{\mu} C_{q}^{\gamma} A_{2(\mu+\gamma)-2}(c,|\boldsymbol{y}|) H_{p+q-2(\mu+\gamma), 2}(\boldsymbol{y}) \\
& +\underbrace{\sum_{\mu=0}^{\frac{p}{2}-1} \sum_{\gamma=0}^{\frac{q-1}{2}}}_{p_{\mu}<q_{\gamma}}(-1)^{\frac{q-1}{2}+\gamma} C_{p}^{\mu} C_{q}^{\gamma} A_{2(p+\gamma-\mu)-2}(c,|\boldsymbol{y}|) H_{q-p-2(\gamma-\mu), 2}(\boldsymbol{y}) \\
& -\underbrace{\sum_{\mu=0}^{\frac{p}{2}-1} \sum_{\gamma=0}^{\frac{q-1}{2}}}_{p_{\mu}>q_{\gamma}}(-1)^{\frac{q-1}{2}+\gamma} C_{p}^{\mu} C_{q}^{\gamma} A_{2(q+\mu-\gamma)-2}(c,|\boldsymbol{y}|) H_{p-q-2(\mu-\gamma), 2}(\boldsymbol{y}) .
\end{aligned}
$$

2.6. $p \geqslant 2$ is even and $q \geqslant 2$ is even, $p+q \geqslant 4$

Using the Fourier series for the boundary monomial

$$
\begin{aligned}
\stackrel{\circ}{R}_{p, q}^{\prime}(\varphi) & =c^{p} \cos ^{p} \varphi c^{q} \sin ^{q} \varphi \\
& =c^{p+q}\left(\frac{C_{p}^{\frac{p}{2}}}{2^{p}}+\frac{1}{2^{p-1}} \sum_{\mu=0}^{\frac{p}{2}-1} C_{p}^{\mu} \cos \varphi_{\mu}\right)\left(\frac{C_{q}^{\frac{q}{2}}}{2^{q}}+\frac{1}{2^{q-1}} \sum_{\gamma=0}^{\frac{q}{2}-1}(-1)^{\frac{q}{2}+\gamma} C_{q}^{\gamma} \sin \varphi_{\gamma}\right) \\
& +\frac{c^{p+q}}{2^{p+q}} C_{p}^{\frac{p}{2}} C_{q}^{\frac{q}{2}} \\
& +\frac{c^{p+q}}{2^{p+q-1}} C_{q}^{\frac{q}{2}} \sum_{\mu=0}^{\frac{p}{2}-1} C_{p}^{\mu} \cos \left(\varphi_{\mu}\right)+\frac{c^{p+q}}{2^{p+q-1}} C_{p}^{\frac{p}{2}} \sum_{\gamma=0}^{\frac{q}{2}-1}(-1)^{\frac{q}{2}+\gamma} C_{q}^{\gamma} \cos \left(\varphi_{\gamma}\right) \\
& +\frac{c^{p+q}}{2^{p+q-1}} \sum_{\mu=0}^{\frac{p}{2}-1} \sum_{\gamma=0}^{\frac{q}{2}-1}(-1)^{\frac{q}{2}+\gamma} C_{p}^{\mu} C_{q}^{\gamma} \cos \left(\varphi_{\gamma}+\varphi_{\mu}\right) \\
& +\frac{c^{p+q}}{2^{p+q-1}} \sum_{\mu=0}^{\frac{p}{2}-1} \sum_{\gamma=0}^{\frac{q}{2}-1}(-1)^{\frac{q}{2}+\gamma} C_{p}^{\mu} C_{q}^{\gamma} \cos \left(\varphi_{\gamma}-\varphi_{\mu}\right)
\end{aligned}
$$


and not extending it explicitly to the disk set up the solution to the problem in polar variables and implement the identical transformation

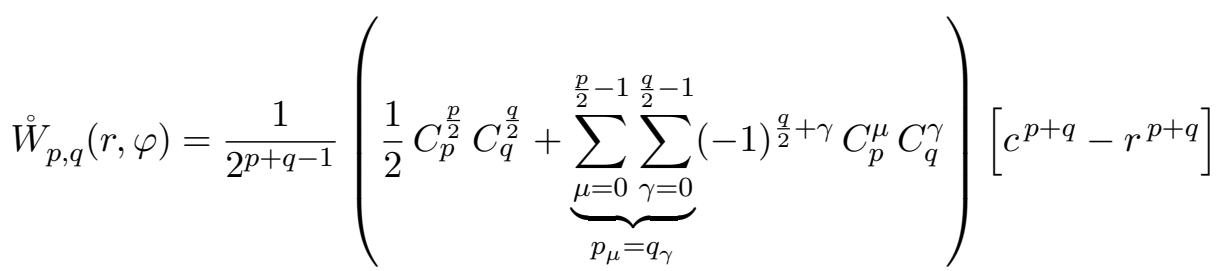

$$
\begin{aligned}
& +\frac{1}{2^{p+q-1}} \sum_{\mu=0}^{\frac{p}{2}-1} C_{p}^{\mu}\left[c^{p+q}\left(\frac{r}{c}\right)^{p_{\mu}}-r^{p+q}\right] \cos \varphi_{\mu} \\
& +\frac{1}{2^{p+q-1}} \sum_{\gamma=0}^{\frac{q}{2}-1}(-1)^{\frac{q}{2}+\gamma} C_{q}^{\gamma}\left[c^{p+q}\left(\frac{r}{c}\right)^{q_{\gamma}}-r^{p+q}\right] \cos \varphi_{\gamma}
\end{aligned}
$$

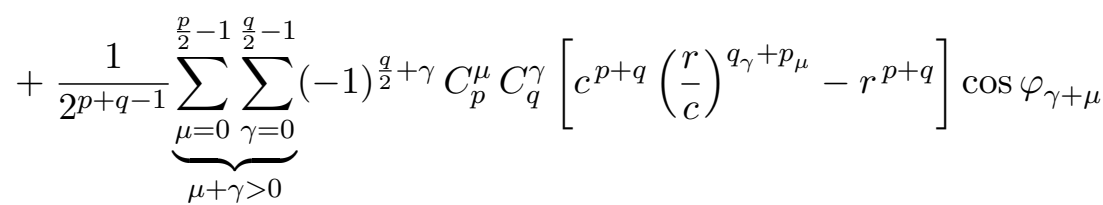

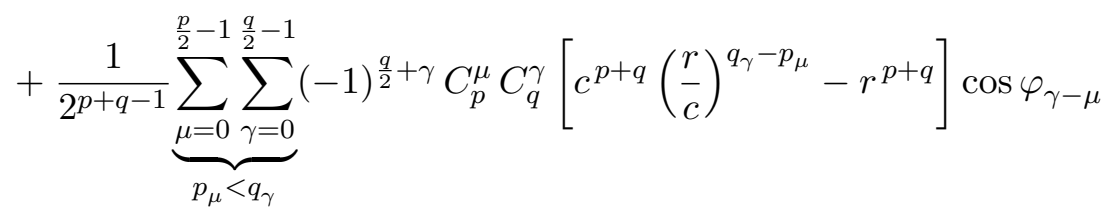

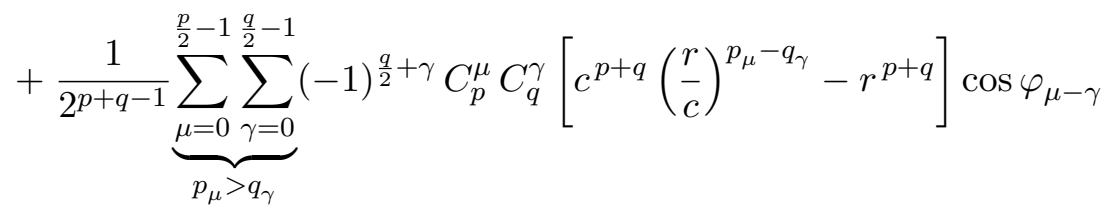

$$
\begin{aligned}
& +\stackrel{\circ}{R}_{p, q}(r, \varphi) \text {. }
\end{aligned}
$$

This case needs much more work compared to previous cases, and eventually we obtain the polynomial $S_{p, q}$ in polar variables 


$$
\begin{aligned}
& 2^{p+q-1} \stackrel{\stackrel{S}{S}}{p, q}_{(r, \varphi)}=(\frac{1}{2} C_{p}^{\frac{p}{2}} C_{q}^{\frac{q}{2}}+\underbrace{\sum_{\mu=0}^{\frac{p}{2}-1} \sum_{\gamma=0}^{\frac{q}{2}-1}}_{p_{\mu}=q_{\gamma}}(-1)^{\frac{q}{2}+\gamma} C_{p}^{\mu} C_{q}^{\gamma}) A_{p+q-2}(r) \\
& +\sum_{\mu=0}^{\frac{p}{2}-1} C_{p}^{\mu} A_{q+2 \mu-2}(r) r^{p_{\mu}} \cos \left(p_{\mu} \varphi\right) \\
& +\sum_{\gamma=0}^{\frac{q}{2}-1}(-1)^{\frac{q}{2}+\gamma} C_{q}^{\gamma} A_{p+2 \gamma-2}(r) r^{q_{\gamma}} \cos \left(q_{\gamma} \varphi\right)
\end{aligned}
$$

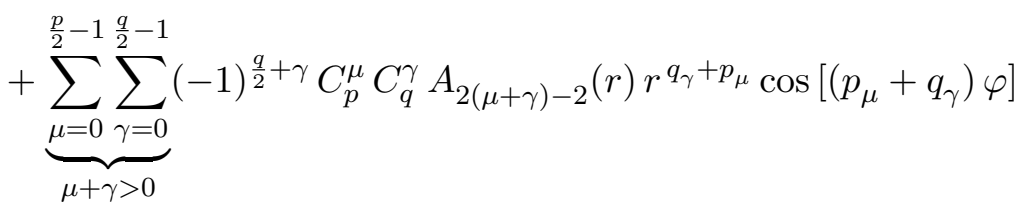

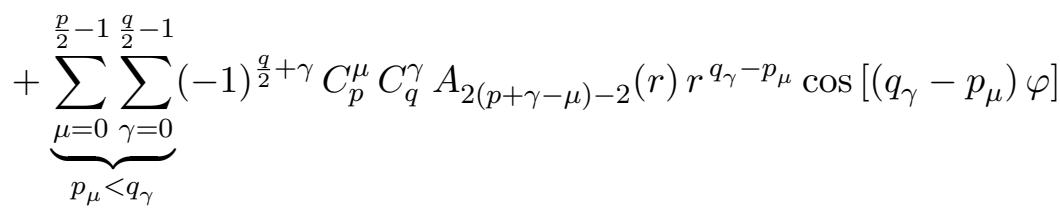

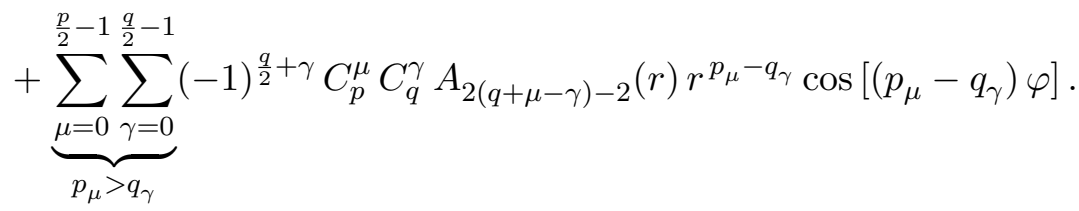

Transforming the polynomial $S_{p, q}$ to cartesian variables is quite routine to obtain 


$$
\begin{aligned}
2^{p+q-1} S_{p, q}(\boldsymbol{y}) & =(\frac{1}{2} C_{p}^{\frac{p}{2}} C_{q}^{\frac{q}{2}}+\underbrace{\frac{p}{2}-1}_{p_{\mu}=q_{\gamma}} \sum_{\gamma=0}^{\frac{q}{2}-1}(-1)^{\frac{q}{2}+\gamma} C_{p}^{\mu} C_{q}^{\gamma}) A_{p+q-2}(|\boldsymbol{y}|) \\
& +\sum_{\mu=0}^{\frac{p}{2}-1} C_{p}^{\mu} A_{q+2 \mu-2}(|\boldsymbol{y}|) H_{p-2 \mu, 1}(\boldsymbol{y}) \\
& +\sum_{\gamma=0}^{\frac{q}{2}-1}(-1)^{\frac{q}{2}+\gamma} C_{q}^{\gamma} A_{p+2 \gamma-2}(|\boldsymbol{y}|) H_{q-2 \gamma, 1}(\boldsymbol{y}) \\
& +\underbrace{\sum_{\mu=0}^{\frac{p}{2}-1} \sum_{\gamma=0}^{\frac{q}{2}-1}(-1)^{\frac{q}{2}+\gamma} C_{p}^{\mu} C_{q}^{\gamma} A_{2(\mu+\gamma)-2}(|\boldsymbol{y}|) H_{p+q-2(\mu+\gamma), 1}(\boldsymbol{y})}_{\mu+\gamma>0} \\
& +\underbrace{\sum_{\mu=0}^{\frac{p}{2}-1} \sum_{\gamma=0}^{\frac{q}{2}-1}(-1)^{\frac{q}{2}+\gamma} C_{p}^{\mu} C_{q}^{\gamma} A_{2(p+\gamma-\mu)-2}(|\boldsymbol{y}|) H_{q-p-2(\gamma-\mu), 1}(\boldsymbol{y})}_{p_{\mu}<q_{\gamma}} \\
& +\underbrace{\sum_{\mu=0}^{\frac{p}{2}-1} \sum_{\gamma=0}^{\frac{q}{2}-1}(-1)^{\frac{q}{2}+\gamma} C_{p}^{\mu} C_{q}^{\gamma} A_{2(q+\mu-\gamma)-2}(|\boldsymbol{y}|) H_{p-q-2(\mu-\gamma), 1}(\boldsymbol{y}) .}_{p_{\mu}>q_{\gamma}}
\end{aligned}
$$

\section{Examples on the Fourier method to find $S_{p, q}(\boldsymbol{y})$}

Here and below we consider the Dirichlet problem (1.1), where $\boldsymbol{x}_{0}=\mathbf{0}$, hence, all the resulted formulas, namely (2.8)-(2.11), are valid when formally replacing variables $\boldsymbol{y}$ with $\boldsymbol{x}$. For readers interested in deriving the explicit formulas for the coefficients of polynomials $S_{p, q}$ we place in Table 1 the Fourier series of all the boundary monomials $\stackrel{\circ}{R}_{p, q}^{\prime}$. The resulted polynomials $S_{p, q}$ are placed in Table 2 and could attract attention of shrewd readers by very high level of packing the independent variables.

For example, the polynomial $S_{6,6}$ is written (in Table 2) using 3 non-zero coefficients (essential, or primary), but admits the following partial expanding 
Table 1. The Fourier series of boundary monomials $\stackrel{\circ}{R}_{p, q}^{\prime}(\varphi)$

\begin{tabular}{|c|c|}
\hline No & The Fourier series \\
\hline 1 & $\stackrel{\circ}{R}_{7,0}^{\prime}=+\frac{35}{64} c^{7} \cos \varphi+\frac{21}{84} c^{7} \cos 3 \varphi+\frac{7}{64} c^{7} \cos 5 \varphi+\frac{1}{64} c^{7} \cos 7 \varphi$ \\
\hline 2 & $\stackrel{\circ}{R}_{0,7}^{\prime}=+\frac{35}{64} c^{7} \sin \varphi-\frac{21}{84} c^{7} \sin 3 \varphi+\frac{7}{64} c^{7} \sin 5 \varphi+\frac{1}{64} c^{7} \sin 7 \varphi$ \\
\hline 3 & $\stackrel{\circ}{R}_{8,0}^{\prime}=+\frac{35}{128} c^{8}+\frac{7}{16} c^{8} \cos 2 \varphi+\frac{7}{32} c^{8} \cos 4 \varphi+\frac{1}{16} c^{8} \cos 6 \varphi+\frac{1}{128} c^{8} \cos 8 \varphi$ \\
\hline 4 & $\stackrel{\circ}{R}_{8,0}^{\prime}=+\frac{35}{128} c^{8}-\frac{7}{16} c^{8} \cos 2 \varphi+\frac{7}{32} c^{8} \cos 4 \varphi-\frac{1}{16} c^{8} \cos 6 \varphi+\frac{1}{128} c^{8} \cos 8 \varphi$ \\
\hline 5 & $\stackrel{\circ}{R}_{3,3}^{\prime}=+\frac{3}{32} c^{6} \sin 2 \varphi-\frac{1}{32} c^{6} \sin 6 \varphi$ \\
\hline 6 & $\stackrel{\circ}{R}_{3,4}^{\prime}=+\frac{3}{64} c^{7} \cos \varphi-\frac{3}{64} c^{7} \cos 3 \varphi-\frac{1}{64} c^{7} \cos 5 \varphi+\frac{1}{64} c^{7} \cos 7 \varphi$ \\
\hline 7 & $\stackrel{\circ}{R}_{4,3}^{\prime}=+\frac{3}{64} c^{7} \sin \varphi+\frac{3}{64} c^{7} \sin 3 \varphi-\frac{1}{64} c^{7} \sin 5 \varphi-\frac{1}{64} c^{7} \sin 7 \varphi$ \\
\hline 8 & $\stackrel{\circ}{R}_{4,4}^{\prime}=+\frac{3}{128} c^{8}-\frac{1}{32} c^{8} \cos 4 \varphi+\frac{1}{128} c^{8} \cos 8 \varphi$ \\
\hline 9 & $\stackrel{\circ}{R}_{3,5}^{\prime}=+\frac{3}{64} c^{8} \sin 2 \varphi-\frac{1}{64} c^{8} \sin 4 \varphi-\frac{1}{64} c^{8} \sin 6 \varphi+\frac{1}{128} c^{8} \sin 8 \varphi$ \\
\hline 10 & $\stackrel{\circ}{R}_{5,3}^{\prime}=+\frac{3}{64} c^{8} \sin 2 \varphi+\frac{1}{64} c^{8} \sin 4 \varphi-\frac{1}{64} c^{8} \sin 6 \varphi-\frac{1}{128} c^{8} \sin 8 \varphi$ \\
\hline 11 & $\stackrel{\circ}{R}_{3,6}^{\prime}=+\frac{3}{128} c^{9} \cos \varphi-\frac{1}{32} c^{9} \cos 3 \varphi+\frac{3}{256} c^{9} \cos 7 \varphi-\frac{1}{256} c^{9} \cos 9 \varphi$ \\
\hline 12 & $\stackrel{\circ}{R}_{6,3}^{\prime}=+\frac{3}{128} c^{9} \sin \varphi+\frac{1}{32} c^{9} \sin 3 \varphi-\frac{3}{256} c^{9} \sin 7 \varphi-\frac{1}{256} c^{9} \sin 9 \varphi$ \\
\hline 13 & $\stackrel{\circ}{R}_{5,5}^{\prime}=+\frac{5}{256} c^{10} \sin 2 \varphi-\frac{5}{512} c^{10} \sin 6 \varphi+\frac{1}{512} c^{10} \sin 10 \varphi$ \\
\hline 14 & $\stackrel{\circ}{R}_{6,6}^{\prime}=+\frac{5}{1024} c^{12}-\frac{15}{2048} c^{12} \cos 4 \varphi+\frac{3}{1024} c^{12} \cos 8 \varphi-\frac{1}{2048} c^{12} \cos 12 \varphi$ \\
\hline
\end{tabular}

$$
\begin{aligned}
2^{11} S_{6,6} & =10\left(c^{10}+c^{8}|\boldsymbol{x}|^{2}+c^{6}|\boldsymbol{x}|^{4}+c^{4}|\boldsymbol{x}|^{6}+c^{2}|\boldsymbol{x}|^{8}+|\boldsymbol{x}|^{10}\right) \\
& -15\left(c^{6}+c^{4}|\boldsymbol{x}|^{2}+c^{2}|\boldsymbol{x}|^{4}+\left.\boldsymbol{x}\right|^{6}\right)\left(x_{1}^{4}-6 x_{1}^{2} x_{2}^{2}+x_{2}^{4}\right) \\
& +6\left(c^{2}+|\boldsymbol{x}|^{2}\right)\left(x_{1}^{8}-28 x_{1}^{6} x_{2}^{2}+70 x_{1}^{4} x_{2}^{4}-28 x_{1}^{2} x_{2}^{6}+x_{2}^{8}\right)
\end{aligned}
$$

Fully expanded polynomial $S_{6,6}$ is given in Table 4 . 
Table 2. Polynomials $S_{p, q}(\boldsymbol{x})$ (arguments $(c,|\boldsymbol{x}|)$ of the homogeneous polynomials $A$ and argument $(\boldsymbol{x})$ of the harmonic polynomials $H$ are not shown for brevity; '\# of terms' in the last column refers to fully expanded polynomials, see Table 4 in Section 7)

\begin{tabular}{|r|l|c|}
\hline No & \multicolumn{1}{|c|}{$S_{p, q}(\boldsymbol{x})$} & \# of terms \\
\hline 1 & $2^{6} S_{7,0}=+35 A_{4} H_{1,1}+21 A_{2} H_{3,1}+7 A_{0} H_{5,1}$ & 6 \\
2 & $2^{6} S_{0,7}=+35 A_{4} H_{1,2}-21 A_{2} H_{3,2}+7 A_{0} H_{5,2}$ & 6 \\
3 & $2^{7} S_{8,0}=+35 A_{6} H_{0,1}+56 A_{4} H_{2,1}+28 A_{2} H_{4,1}+8 A_{0} H_{6,1}$ & 10 \\
4 & $2^{7} S_{0,8}=+35 A_{6} H_{0,1}-56 A_{4} H_{2,1}+28 A_{2} H_{4,1}-8 A_{0} H_{6,1}$ & 10 \\
5 & $2^{5} S_{3,3}=+3 A_{2} H_{2,2}$ & 3 \\
6 & $2^{6} S_{3,4}=+3 A_{4} H_{1,1}-3 A_{2} H_{3,1}-A_{0} H_{5,1}$ & 5 \\
7 & $2^{6} S_{4,3}=+3 A_{4} H_{1,2}+3 A_{2} H_{3,2}-A_{0} H_{5,2}$ & 5 \\
8 & $2^{7} S_{4,4}=+3 A_{6} H_{0,1}-4 A_{2} H_{4,1}$ & 10 \\
9 & $2^{7} S_{3,5}=+6 A_{4} H_{2,2}-2 A_{2} H_{4,2}-2 A_{0} H_{6,2}$ & 6 \\
10 & $2^{7} S_{5,3}=+6 A_{4} H_{2,2}+2 A_{2} H_{4,2}-2 A_{0} H_{6,2}$ & 6 \\
11 & $2^{8} S_{3,6}=+6 A_{6} H_{1,1}-8 A_{4} H_{3,1}+3 A_{0} H_{7,1}$ & 10 \\
12 & $2^{8} S_{6,3}=+6 A_{6} H_{1,2}+8 A_{4} H_{3,2}-3 A_{0} H_{7,2}$ & 10 \\
13 & $2^{9} S_{5,5}=+10 A_{6} H_{2,2}-5 A_{2} H_{6,2}$ & 21 \\
14 & $2^{11} S_{6,6}=+10 A_{10} H_{0,1}-15 A_{6} H_{4,1}+6 A_{2} H_{8,1}$ & 10 \\
\hline
\end{tabular}

\section{Some improvements to find $S_{p, q}(\boldsymbol{y})$}

From the explicit formulas (2.8)-(2.11) derived in Section 2 and the examples considered in Section 3 one could deduce that the contribution of a monomial $R_{p, q}(\boldsymbol{y})$ to the resulted polynomial $S_{m-2}(\boldsymbol{y})(1.9)$ is given as

$$
2^{p+q-1} S_{p, q}(\boldsymbol{y})=\sum_{\rho=0}^{\frac{p+q-1}{2}} A_{2 \rho}(c,|\boldsymbol{y}|)\left(\alpha_{2 \rho} H_{p+q-2-2 \rho, 1}(\boldsymbol{y})+\sigma_{2 \rho} H_{p+q-2-2 \rho, 2}(\boldsymbol{y})\right)
$$

when $p+q$ is odd, and as

$$
2^{p+q-1} S_{p, q}(\boldsymbol{y})=\sum_{\rho=0}^{\frac{p+q}{2}-1} A_{2 \rho}(c,|\boldsymbol{y}|)\left(\alpha_{2 \rho} H_{p+q-2-2 \rho, 1}(\boldsymbol{y})+\sigma_{2 \rho} H_{p+q-2-2 \rho, 2}(\boldsymbol{y})\right)
$$


when $p+q$ is even, where the coefficients $\alpha_{2 \rho}, \sigma_{2 \rho}$ are fully determined by the Fourier series of the monomial $R_{p, q}^{\prime}(\boldsymbol{y})$ through formulas (2.8)-(2.11).

We show here how to simplify the computation of the coefficients $\alpha_{2 \rho}, \sigma_{2 \rho}$.

Proposition 4.1. The Fourier series of the Laplacian of a monomial $R_{p, q}(\boldsymbol{y})$, i. e. function $\Delta_{\boldsymbol{y}} R_{p, q}(\boldsymbol{y})$ restricted to $\mathcal{S}_{c}^{2}(\mathbf{0})$, fully determines (the coefficients $\alpha_{2 \rho}, \sigma_{2 \rho}$ of) the polynomial $S_{p, q}(\boldsymbol{y})$.

Proof. First, we derive a partial differential equation the polynomial $S_{p, q}(\boldsymbol{y})$ satisfies. For this we substitute representation (1.7) into the differential equation of the problem (1.4)

$$
\Delta_{\boldsymbol{y}}\left(\left(c^{2}-|\boldsymbol{y}|^{2}\right) S_{p, q}\right)=-\Delta_{\boldsymbol{y}} R_{p, q}
$$

to obtain

$$
\left(c^{2}-|\boldsymbol{y}|^{2}\right) \Delta_{\boldsymbol{y}} S_{p, q}-4\left(y_{1} \frac{\partial S_{p, q}}{\partial y_{1}}+y_{2} \frac{\partial S_{p, q}}{\partial y_{2}}+S_{p, q}\right)=-\Delta_{\boldsymbol{y}} R_{p, q}
$$

a degenerate elliptic linear partial differential equation with variable coefficients.

Second, when $|\boldsymbol{y}| \rightarrow c$, the higher order terms of (4.4) vanish, and we obtain the following Robin boundary condition

$$
\left.4\left(r \frac{\partial \stackrel{\circ}{S}_{p, q}(r, \varphi)}{\partial r}+\stackrel{\circ}{S}_{p, q}(r, \varphi)\right)\right|_{r=c}=\left.\Delta_{(r, \varphi)} \stackrel{\circ}{R}_{p, q}(r, \varphi)\right|_{r=c}
$$

written in polar variables.

Third, substituting representation (4.1) or (4.2) for the polynomial $S_{p, q}(\boldsymbol{y})$ on the left-hand side of the boundary condition (4.5) we obtain a trigonometric polynomial, whereas the right-hand side of (4.5) is easily reduced due to formulas (..) to the Fourier series of function $\Delta_{y} R_{p, q}(\boldsymbol{y})$ restricted to $\mathcal{S}_{c}^{2}(\mathbf{0})$.

Comparing the respective coefficients of both trigonometric polynomials we easily find simple algebraic relations between the unknown coefficients of the polynomial $S_{p, q}(\boldsymbol{y})$ and the Fourier series of function $\Delta_{\boldsymbol{y}} R_{p, q}(\boldsymbol{y})$ restricted to $\mathcal{S}_{c}^{2}(\mathbf{0})$. For the sake of brevity we do not derive here the proper relations and leave this derivation to shrewd readers.

Since the Dirichlet problem (1.4) is linear it immediately follows from proposition 4.1 the following

Proposition 4.2. The Fourier series of the Laplacian of the boundary polynomial $R_{m}(\boldsymbol{y})$, i. e. function $\Delta_{\boldsymbol{y}} R_{m}(\boldsymbol{y})$ restricted to $\mathcal{S}_{c}^{2}(\mathbf{0})$, fully determines the polynomial $S_{m-2}(\boldsymbol{y})$. 


\section{Examples on improvements to find $S_{p, q}(\boldsymbol{y})$}

For readers interested in deriving the explicit formulas for the coefficients of polynomials $S_{p, q}(\boldsymbol{y})$ we place in Table 3 the Fourier series of the Laplacians of monomials $R_{p, q}$, where the monomials $R_{p, q}$ are the same as in Table 1 . The Fourier series in Table 3 supplemented with the boundary condition (4.5) fully determine the polynomials $S_{p, q}$ in Table 2 .

Table 3. The Fourier series of functions $\Delta_{\boldsymbol{x}} R_{p, q}(\boldsymbol{x})$ restricted to $\mathcal{S}_{c}^{2}(\mathbf{0})$

\begin{tabular}{|l|l|}
\hline No & \multicolumn{1}{|c|}{ The Fourier series } \\
\hline 1 & $\left(\Delta_{x} R_{7,0}\right)^{\prime}=+\frac{105}{4} c^{5} \cos \varphi+\frac{105}{8} c^{5} \cos 3 \varphi+\frac{21}{8} c^{5} \cos 5 \varphi$ \\
3 & $\left(\Delta_{x} R_{0,7}\right)^{\prime}=+\frac{105}{4} c^{5} \sin \varphi-\frac{105}{8} c^{5} \sin 3 \varphi+\frac{21}{8} c^{5} \sin 5 \varphi$ \\
4 & $\left(\Delta_{x} R_{8,0}\right)^{\prime}=+\frac{35}{2} c^{6}+\frac{105}{4} c^{6} \cos 2 \varphi+\frac{21}{2} c^{8} \cos 4 \varphi+\frac{7}{4} c^{6} \cos 6 \varphi$ \\
5 & $\left(\Delta_{x} R_{0,8}\right)^{\prime}=+\frac{35}{2} c^{6}-\frac{105}{4} c^{6} \cos 2 \varphi+\frac{21}{2} c^{8} \cos 4 \varphi-\frac{7}{4} c^{6} \cos 6 \varphi$ \\
6 & $\left(\Delta_{x} R_{3,3}\right)^{\prime}=+3 c^{4} \sin 2 \varphi$ \\
7 & $\left(\Delta_{x} R_{3,4}\right)^{\prime}=+\frac{9}{4} c^{5} \cos \varphi-\frac{15}{8} c^{5} \cos 3 \varphi-\frac{3}{8} c^{5} \cos 5 \varphi$ \\
8 & $\left(\Delta_{x} R_{4,3}\right)^{\prime}=+\frac{9}{4} c^{5} \sin \varphi+\frac{15}{8} c^{5} \sin 3 \varphi-\frac{3}{8} c^{5} \sin 5 \varphi$ \\
9 & $\left(\Delta_{x} R_{4,4}\right)^{\prime}=+\frac{3}{2} c^{6}-\frac{3}{2} c^{6} \cos 4 \varphi$ \\
10 & $\left(\Delta_{x} R_{3,5}\right)^{\prime}=+\frac{45}{16} c^{6} \sin 2 \varphi-\frac{3}{4} c^{8} \sin 4 \varphi-\frac{7}{16} c^{6} \sin 6 \varphi$ \\
11 & $\left(\Delta_{x} R_{5,3}\right)^{\prime}=+\frac{45}{16} c^{6} \sin 2 \varphi+\frac{3}{4} c^{8} \sin 4 \varphi-\frac{7}{16} c^{6} \sin 6 \varphi$ \\
12 & $\left(\Delta_{x} R_{3,6}\right)^{\prime}=+\frac{15}{8} c^{7} \cos \varphi-\frac{9}{4} c^{7} \cos 3 \varphi+\frac{3}{8} c^{7} \cos 7 \varphi$ \\
$\left(\Delta_{x} R_{6,3}\right)^{\prime}=+\frac{15}{8} c^{7} \sin \varphi+\frac{9}{4} c^{7} \sin 3 \varphi-\frac{3}{8} c^{7} \sin 7 \varphi$ \\
$\left(\Delta_{x} R_{5,5}\right)^{\prime}=+\frac{15}{8} c^{8} \sin 2 \varphi-\frac{5}{8} c^{8} \sin 6 \varphi$ \\
$\left(\Delta_{x} R_{6,6}\right)^{\prime}=+\frac{45}{64} c^{10}-\frac{15}{16} c^{10} \cos 4 \varphi+\frac{15}{64} c^{10} \cos 8 \varphi$
\end{tabular}




\section{Some other methods to find $S_{p, q}$}

Suppose we know that representation (1.6) holds for the solution $W_{m}(\boldsymbol{y})$ to the Dirichlet problem (1.4). Then we can find polynomial $S_{p, q}(\boldsymbol{y})$ using a lot of methods different from that developed in Section 2.

First, we can use the degenerate elliptic partial differential equation (4.4). This equation needs no boundary condition to be solved, hence, the method of undetermined coefficients is applicable.

Second, for solving the Poisson differential equation (4.3) with zero boundary values the Ritz method [6,7] ideally suits.

Third, for solving the above problem for the Poisson differential equation the Green integral formula is applicable, where the Green function is given in [5].

The above methods give required polynomials through a huge bulk of computational work, contrary to the method of Section 2 or its improved version of Section 4 . In other words, the above methods are implicit ones.

\section{Examples on other methods to find $S_{p, q}$}

Polynomials $S_{p, q}$ obtained by any method of Section 6 are fully expanded. Some of them are placed in Table 4.

Table 4 . Some fully expanded polynomials $S_{p, q}(\boldsymbol{x})$

\begin{tabular}{|c|c|}
\hline No & $S_{p, q}(\boldsymbol{x})$ \\
\hline 1 & $2^{6} S_{7,0}=+63 x^{5}-42 x^{3} y^{2}+7 x y^{4}+56 c^{2} x^{3}-28 c^{2} x y^{2}+35 c^{4} x$ \\
\hline 4 & $2^{7} S_{0,8}=-x^{6}+29 x^{4} y^{2}-99 x^{2} y^{4}+127 y^{6}$ \\
\hline & $+7 c^{2} x^{4}-98 c^{2} x^{2} y^{2}+119 c^{2} y^{4}-21 c^{4} x^{2}+91 c^{4} y^{2}+35 c^{6}$ \\
\hline 9 & $2^{7} S_{3,5}=-8 x^{5} y+64 x^{3} y^{3}+8 x y^{5}+4 c^{2} x^{3} y+20 c^{2} x y^{3}+12 c^{4} x y$ \\
\hline 14 & $2^{11} S_{6,6}=+x^{10}-67 x^{8} y^{2}+562 x^{6} y^{4}+562 x^{4} y^{6}-67 x^{2} y^{8}+y^{10}$ \\
\hline & $+c^{2} x^{8}-68 c^{2} x^{6} y^{2}+630 c^{2} x^{4} y^{4}-68 c^{2} x^{2} y^{6}+c^{2} y^{8}$ \\
\hline & $-5 c^{4} x^{6}+105 c^{4} x^{4} y^{2}+105 c^{4} x^{2} y^{4}-5 c^{4} y^{6}$ \\
\hline & $-5 c^{6} x^{4}+110 c^{6} x^{2} y^{2}-5 c^{6} y^{4}+10 c^{8} x^{2}+10 c^{8} y^{2}+10 c^{10}$ \\
\hline
\end{tabular}




\section{Conclusions}

1. An explicit solver for the Dirichlet problem for the Laplace equation in a disk in polynomials is presented.

2. The solver essentially uses representation (1.3) for the solution to the problem but admits very high level of packing independent variables.

3. The level of packing independent variables is influenced by the number of essential coefficients of the solution.

4. The number of essential coefficients of the solution equals the number of nonzero Fourier coefficients of function $\Delta R_{m}$ restricted to the boundary of the disk, where $R_{m}$ is the boundary polynomial.

\section{Acknowledgements}

We express our sincere gratefulness to Prof. P. K. Kogut for his assistance and support during submitting the article.

\section{References}

1. V. L. Borsch, I. E. Platonova, On a representation of the solution to the Dirichlet problem in a circle, Visnyk DNU, Series: Mathematical Modelling, Dnepropetrovsk: DNU, 24(9)(2017), 1-18.

2. V. L. Borsch, I. E. Platonova, On a representation of the solution to the Dirichlet problem in a disk. The Poisson integral based solution in polynomials, JODEA, 26(1)(2018), $72-77$.

3. É. Goursat, Cours d'Analyse Mathématique: Tome 3, Gauthier-Villars, Paris, 1915. (Cours de la Faculté des Sciences de Paris)

4. I. S. Gradshteyn, I. M. Ryzhik, Table of Integrals, Series, and Products: translated from Russian, Academic Press, Amsterdam, 2007.

5. L. V. Kantorovich, V. I. Krylov, Approximate Methods of Higher Analysis: translated from Russian, Interscience, NY, 1959.

6. S. G. Mikhlin, Variational Methods in Mathematical Physics: translated from Russian, Pergamon Press, Oxford, 1963.

7. K. Rektorys, Variational Methods in Mathematics, Science and Engineering: translated from the Czech, D. Reidel Publishing Company, Boston, 1980.

8. P. K. Suetin, Orthogonal Polynomials in Two Variables: translated from Russian, Taylor \& Francis, Ltd, London, 1999. 